\title{
A New Look at Geyser Formation in Sewer Systems
}

\author{
Steven J. Wright \\ University of Michigan, Ann Arbor, Michigan
}

\begin{abstract}
Previous analysis of pressure and velocity data collected at a site experiencing stormwater geysers suggested a mechanism for the occurrence. Laboratory experiments attempting to reproduce the geysers were promising but inconclusive due to potential scale effects in the small scale experiments. Subsequent researchers have generally used this early work as the starting point for subsequent laboratory experiments. Over the years, a number of videos of geysers in actual systems have been obtained and a review of important features from these videos calls into question certain aspects of the original hypothesis. An alternative explanation for the phenomenon has been developed and an experimental system has been identified that may help resolve some questions. However, laboratory experiments may also be impacted by scale effects and several preliminary studies must be conducted to determine whether there is a fruitful path forward in advancing our knowledge of this phenomenon.
\end{abstract}

\section{Introduction}

The phenomenon commonly referred to as sewer geysering, in which strong jets of an air-water mixture are propelled up through aeration chambers or vertical access shafts, has been intriguing for several decades. Since geysering occurs only infrequently, there has been relatively little documentation of the event. The ready availability of cellular phones with cameras has increased the opportunistic recording of geyser events in recent years and many of these citizen science videos are available on the internet. An official effort by the Minnesota Department of Transportation to document a location that had experienced multiple sewer geysers led to the publishing of one spectacular video on YouTube and other internet sites. Persistence in requesting access to velocity and pressure data that was also collected along with the videos resulted in data for one event that was evaluated (Wright et al. 2011a). An explanation for the formation of sewer geysers was developed (e.g. Wright 2013) and laboratory experiments were performed to demonstrate that explosive ejection of water could occur due to the release of a trapped air pocket in a surcharged conduit without attempting to reproduce any specific geometry of systems where geysers have been observed in actual systems. These experiments confirm that the laboratory geysers also do not involve significant system pressures. However, the laboratory experiments do not quite reproduce what is seen in the available videos and it was hypothesized that this may be due in large part to scale effects with the much smaller scale laboratory experiments, as reported in Wright et al. (2011a) for the prototype geyser. Additional laboratory studies have been more recently performed with the majority of these reproducing the general features of the initial experimental configuration although there is at least one study that reproduces the specific geometry of one location where geysers have been observed on multiple occa- sions. This manuscript presents a critical evaluation of key features of more than 30 geysers that have been recorded on video or otherwise documented in some detail. A modified view of the conditions required for geyser formation is proposed and a revised experimental configuration is proposed to explore a simple manner through which geysers may develop. Small scale laboratory experiments may still be subject to scale effects due to enhanced influence of surface tension effects at the laboratory scale.

Increasing knowledge of this intriguing phenomenon is important in part to increase awareness in the design of large scale sewer interceptors and storage tunnels. Some studies have focused on methods to mitigate sewer geysers that may have drastic consequences on the structural integrity of sewer systems (e.g. Huang 2017; Leon et al. 2019).

\section{Background}

\subsection{Video records of geysers}

Although there are earlier references to ventilation problems in sewer interceptors, often at locations of dropshafts, the phenomenon of geysering became more recognized following the construction of the deep storage tunnels in Chicago's Tunnel and Reservoir Plan (TARP) in the 1980s. The issue of air ventilation was apparently not considered during the design and several sewer geysers were experienced at different locations in the system. A study to investigate the issue was initiated by Guo and Song (1990) and others; Guo and Song (1991) also included a poor resolution photograph of a 1987 geyser in Minneapolis, Minnesota. A major result of this work involved the development of a numerical model (Cardle and Song 1988) to simulate the mixed flow conditions in a rapidly filling conduit. The model did not con-sider the effect of air in the flow dynamics (a continuing

Wright, Steven J. 2021. "A New Look at Geyser Formation in Sewer Systems." Journal of Water Management Modeling 29: C478.

https://doi.org/10.14796/JWMM.C478

(C) Wright 2021. www.chijournal.org ISSN 2292-6062. 
deficiency with almost all more recent models) and this was justified by Guo (1989) with the statement "if the water level rises above the ground surface, the geyser occurs. It has been ascertained that if the dropshaft is ventilated, as most are, the cover could not be blown off by air pressure alone. That is, most blowoffs are caused by the impact forces of the rising water. Therefore, it is sufficient to study the hydrodynamics alone." The basis for this statement is unclear and points to the need for a clear definition of a sewer geyser. In this manuscript, the term is reserved for a condition where a mixture of air and water is ejected from a vertical shaft, rises for meters to tens of meters into the air and is maintained for at least a few seconds, but typically longer.

Examples of sewer geysers that satisfy these criteria are shown in Figure 1.
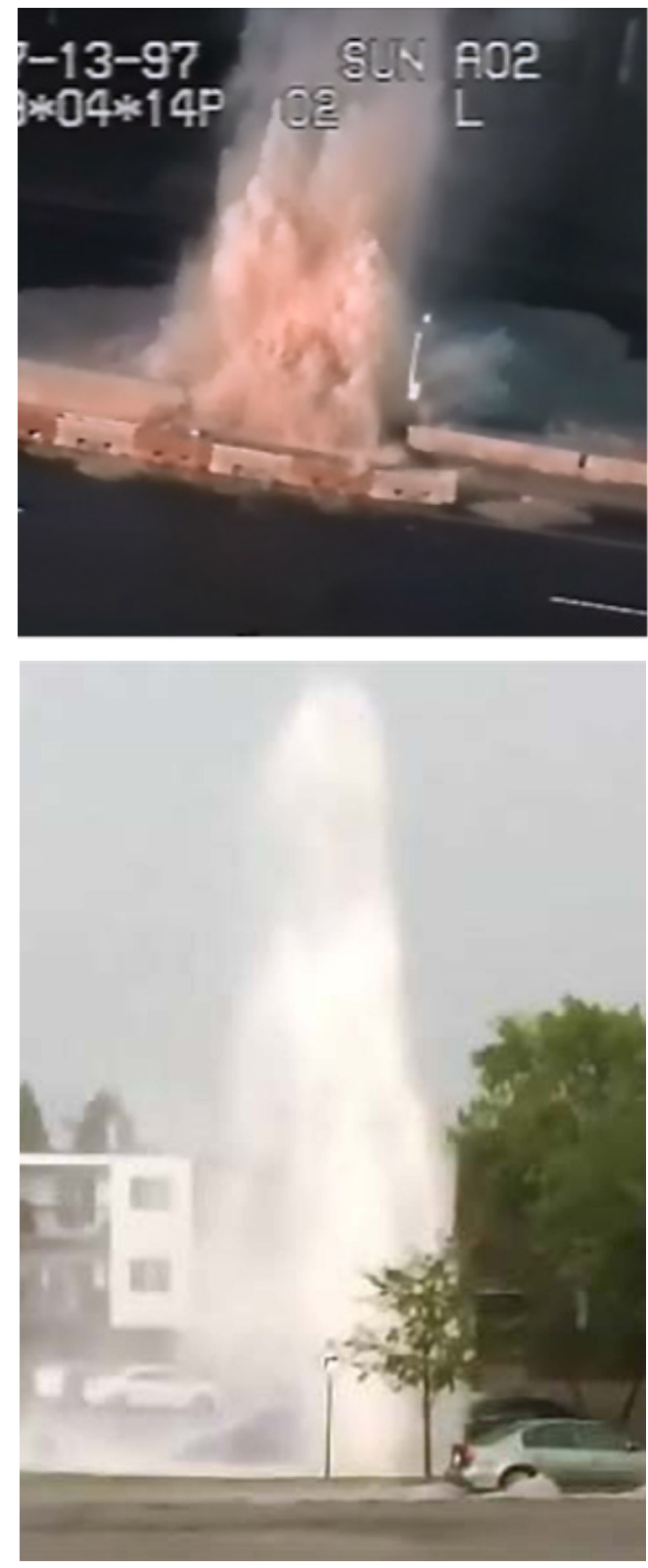

Figure 1 Images of sewer geysers observed in Minneapolis, Minnesota (upper) and Edmonton, Alberta (lower).
A few years later, a stormwater tunnel in Minneapolis, Minnesota that partially followed the alignment of Interstate 35W experienced a repeated series of geysers in at least two locations. The Minnesota Department of Transportation commissioned a study by the University of Minnesota to record velocities, pressures and above ground video at a manhole at the 35th St intersection with $135 \mathrm{~W}$. The group recorded on the order of 20 events from about 1993 to 2006. A 4 min video from one spectacular event on 1999-07-03 has appeared in several YouTube links (e.g. https://www.youtube.com/watch?v=Jp7zLbBs0Rc); this video has sometimes been mistakenly attributed to other cities. Videos on a few other dates are less frequently available and these include only one or two geysers out of several that occurred during the rainfall event. It was subsequently discovered that pressure and velocity measurements were associated with most of the videos, but that the Department of Transportation was reluctant to release the data. Eventually, data from an event on 2004-0711 was provided, analyzed and reported by Wright et al. (2011a, 2011 b) which includes a tentative hypothesis for the mechanisms responsible for geyser formation. This specific event consisted of 9 distinct geysers, each typically lasting about 20 s, spaced in time by $\sim 80$ s. Furthermore, in opposition to the often-expressed view, pressures were not excessive, not nearly sufficient to lift water to the ground surface under hydrostatic conditions and decreased during the geyser event. Unofficially accessed data from a few other events at the same location indicates that these patterns are repeated in other geyser events with the major difference associated with the time interval between geysers apparently being longer when the flow velocity in the tunnel is higher.

In response to the publication of these manuscripts, various other videos of geysers in several other locations have been forwarded to the writer and a review of YouTube is occasionally performed to look for more recently published videos. Video records or other documentation from 34 locations have currently been assembled with various levels of detail. These do not include videos that show a surcharged system from which a manhole cover has been displaced and water is flowing to the ground surface; these are quite easy to distinguish from air-water geysers. They also do not include records with what appears to be basically air (often accompanied by some mist) being discharged. Finally, they do not include geysers for which the video record is $<15 \mathrm{~s}$. Several videos show a single geyser lasting for the duration of the recording while others show the beginning and end of more than one geyser. Note that individual geyser events in the same video are easily distinguishable and the issue is not one of fluctuating rise heights. Burridge and Hunt (2013), for example, discuss that fountains with constant discharge also display fluctuations in the vertical extent of the water jet. These events are not in sewer systems but have somewhat similar characteristics. In almost all cases, no details of the sewer system generating the geyser are available. Some statistics associated with salient features of the geysers are provided below. 


\subsection{Laboratory studies}

So far as is known, the first reporting of a systematic laboratory study on sewer geysers was Wright et al. (2007). Two sets of experiments were designed based on observations during previous experiments that displacement of an air pocket through a vertical riser would exhibit two geyser-like occurrences, one associated with the arrival of the leading edge (based on the earlier experiments described in Vasconcelos and Wright 2011) and the other with the trailing edge of the migrating air pocket. Lewis and Wright (2012) revised the experimental setups and concluded that the trailing edge experiments were essentially a liquid surge problem and therefore not directly relevant to the sewer geyser phenomenon. Although these experiments and later modifications provided some insight, it was after the receipt of the Minnesota data that the leading front experiments were reconfigured to demonstrate the ability to reproduce pressure histories with similar characteristics to those measured during the I35W geysers. The new setup was adjusted a few times to avoid some complicating effects due to the experimental procedure. The final configuration is shown in Figure 2 and results were reported in Wright (2013). These experiments were not intended to model flow in an actual sewer system but were designed to demonstrate geyser-like events where minimal pressures were experienced in the system (i.e. insufficient to lift water to the top of the vertical riser by hydrostatic effects), in part to dispel repeated assertions that geysers were associated with explosive pressures. The water-filled portion of the pipe was maintained in a surcharged condition by means of a constant pressure reservoir, and air was maintained in an isolated portion of pipe at the same pressure. After opening the isolation valve, the air pocket propagated through the otherwise still fluid and escaped due to buoyancy through the vertical shaft. Water pushed ahead of the rising air created the appearance of a geyser. The strength of the geyser as measured by vertical rise above the static level was dependent on several parameters including air pocket volume and the ratio of riser to pipe diameter. Pressure traces resembling those in the Minnesota geysers were measured at the base of the riser. Wright et al. (2017) provide a summary of various air-water interaction processes that can occurring in a rapidly filling sewer system.

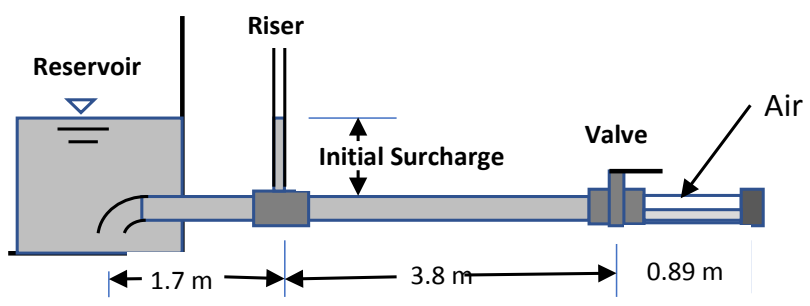

Figure 2 Experimental setup for experiments reported in Wright (2013).

Several other laboratory studies have been conducted since that time; most of them (Chan et al. 2018; Muller et al. 2017; Leon et al. 2019; Wang et al. 2019) involve basically the same type of experimental configuration and, not surprisingly, similar qualitative results were obtained. One issue is that these experiments, following Vasconcelos and Wright (2011), do not equalize the initial pressure across the isolation valve at the beginning of the experiment, resulting in inertial oscillations in the riser that are a result of the experimental configuration but have no connection to flow in actual sewer systems. Huang et al. (2017) perform experiments in a flowing pipeline with a smaller slope downstream so that a stationary hydraulic jump developed downstream from the vertical riser. Increasing the flow rate caused the jump to migrate upstream; this resembles the second type of experiment performed by Wright et al. (2007). In contrast, Liu et al. (2020) modeled the geometry of a specific location in the Edmonton, Alberta sewer system that has occasionally experienced geysers. The configuration involved a small diameter riser at the top of a larger junction chamber in which the inflow would produce an air-water froth for some flow conditions and this froth was forced up through the riser. Three different types of experiment were conducted until the setup produced a sequence of geyser-like events, leading to the conclusion that the movement of an upstream trapped air pocket was a necessary feature of the geysers. Various aspects of this study are discussed in following sections. It should be noted that the laboratory studies demonstrated geyser-like ejections with short period oscillations on the order of $1 \mathrm{~s}$, whereas the prototype geysers exhibit durations of several minutes. This cannot be accounted for simply by the reduced model scale and is likely associated with the short lengths of sewer modeled which set up short period inertial oscillations that would not be present in the much longer prototype system. Finally, a YouTube video was produced as part of a class project at Kansas State University that attempted to explain the Minnesota geysers. This experiment involved flow in a pipe with small amounts of air in the flow and a vertical riser partway along the pipe. The surges in the riser were generated by suddenly closing a valve at the downstream end of the pipe, creating a quasi-water hammer situation that has nothing to do with the sewer geysers discussed above.

\subsection{Numerical simulation studies}

Since the geyser phenomenon is inherently a three-dimensional two-phase flow process, various researchers have attempted to develop models using computational fluid dynamics (CFD) code to simulate the geysering process. Shao and Yost (2018) performed a two-dimensional CFD analysis of the flow in the riser only and imposed a boundary condition at the bottom of the riser. This approach could not include the movement of an entrapped pocket of air in the nearly horizontal sewer, and somewhat arbitrary boundary conditions had to be imposed to obtain predictions that could be compared to the Minnesota geysers. Cataño-Lopera et al. (2014) modeled an event in the TARP system where a geyser was observed at the upstream end of one branch of the system. They used one-dimensional analysis (Leon et al. 2011) to analyze the filling from the downstream end of the branch up to a location just downstream from the geyser 
location and then used this result as a boundary condition for a CFD simulation of a short length of the tunnel and the riser. Although the filling process forced air back upstream through the riser and was almost certainly the cause of the geyser, the simulation results had little resemblance to the observed characteristics of the geyser. One relevant feature was that the riser served as a dropshaft and the falling water from near the ground surface at that location was an essential component of the prediction. Qian et al. (2020) performed a CFD analysis of the laboratory model of the Edmonton geyser and could predict a surge through the vertical riser but it was noted to be unable to reproduce the details of the laboratory surges. This was attributed to the inability of the numerical model to represent the nature of the air-water dynamics in the large air content state. It is concluded that the application of traditional CFD models that essentially model the liquid phase with a small air fraction is unlikely to provide much insight into the geyser phenomenon.

\section{Assessment of existing data}

\subsection{Another view of the Minnesota geysers}

The analysis of the pressure and velocity data of the Minnesota geysers by Wright et al. (2011b) indicated that the 2004-07-11 event involved surcharge conditions in the stormwater tunnel of at most $1.2 \mathrm{~m}$ during non-geyser conditions, which could not have raised the water level in the manhole to the ground surface $27.4 \mathrm{~m}$ above the tunnel invert, let alone an additional $20 \mathrm{~m}$ into the air as estimated from the associated video. Furthermore, the pressure at the tunnel crown dropped down to just above atmospheric pressure during the geyser events. It was concluded that the geyser would have been a low density mixture of air and water with an estimated $4 \%$ water content in order to propel the geyser to the observed height. The original analysis suggested that a Taylor bubble (Davies and Taylor 1950) rising in the manhole would shear off water droplets from the downflowing water around the manhole circumference that would then be carried upwards, creating the air-water mixture. However, a problem with that hypothesis is that all of the surcharged water in the access chamber should have been ejected in about $1.5 \mathrm{~s}$ in an admixture of air and water with $\sim 4 \%$ liquid content and the estimated vertical velocity to raise the geyser $20 \mathrm{~m}$ above the ground surface. Laboratory experiments also indicate that a considerable fraction of the water in the vertical riser is pushed ahead of the rising air, thereby reducing further the time required to remove the water initially existing in the surcharged riser. The laboratory experiments by Leon et al. (2019) began with the riser initially filled with water and they report that a series of 3-8 eruptions occurred over $2 \mathrm{~s}-10 \mathrm{~s}$, or at a $\sim 1$ s interval between successive eruptions. In contrast, the Minnesota geysers lasted on the order of $20 \mathrm{~s}$ with $80 \mathrm{~s}$ intervals between successive geysers, suggesting that a different mechanism must be in place. As described in the following section, other geyser videos indicate individual geysers lasting >1 min. The laboratory experiments of Liu et al. (2020) also cast doubt on the interpretation that initial water in the shaft is the source of the geyser and that the geyser is a consequence of this water being forced upwards by rising air released through the vertical shaft.

\subsection{Summary of video observations}

It is likely that different physical configurations exist in the various videos and contribute to the observed differences in the geyser characteristics. For example, there are two videos that technically are not sewer geysers but occur in systems that are used to inject stormwater into underground karst formations (caves) in Florida (personal communication). These injections are through a vertical shaft and one of the videos clearly shows a strong air core vortex forming in the vertical shaft due to the injection, suggesting that air is pulled down into the underground formation that subsequently returns to the ground surface in the form of a geyser. The video for the Keystone injection well shows a continuous geyser from about $36 \mathrm{~s}$ into the video and weakening towards the end of the video at about $2 \mathrm{~min}$. On the other hand, the Lake Holden injection well video captures a series of individual geysers lasting only $\sim 2 \mathrm{~s}$ and spaced $\sim 10 \mathrm{~s}$ apart. Similar variations in geyser strength and duration were observed in the videos of sewer geysers. As mentioned above, a total of 27 videos of geyser events, including the two stormwater injection wells, have been collected. Consider a geyser duration of $10 \mathrm{~s}$ as an arbitrary cutoff between short and long duration geysers. Of the 27 videos, only 3 showed geysers with a duration $<10 \mathrm{~s}$ and the remaining 24 show geysers lasting $>15 \mathrm{~s}$, lasting to $>1 \mathrm{~min}$. In many cases, the estimated duration is controlled by the duration of the video as there are many video clips that show a geyser throughout the entire length of the video clip including several that were $\sim 1 \mathrm{~min}$ in duration. $\mathrm{A}$ reasonable conclusion is that the specific geometry of the system has a major influence on the geyser duration as the four different records available for the Minnesota system all show geysers with a duration of $\sim 20 \mathrm{~s}$, regardless of the strength of the geyser. These have the appearance of a geyser release of a significant volume of air and that the geyser is maintained through the duration of the air release. For example, using the above estimates for the Minnesota geysers, an individual geyser may release on the order of $1800 \mathrm{~m}^{3}$ air. Most of the other videos suggest more modest air volumes, but a few, including the Edmonton geyser referenced by Liu et al. (2020) may indicate comparable air volumes. Analyses of a few relatively large systems have suggested that trapping of air volumes of that same order occurred without the formation of a sewer geyser but with other system damage. It is stressed that the specific system geometry is probably crucial to the occurrence of sewer geysers.

\section{A new interpretation}

\subsection{Essential features}

Interpretation of serendipitous videos is hampered by the lack of information on the specific system geometry. Even with know- 
ledge of geometry, it is typically impossible to reproduce a potentially relevant portion of an interceptor or storage tunnel at any reasonable scale. If the estimate of a trapped air volume on the order of $1800 \mathrm{~m}^{3}$ is reasonable for the Minnesota geysers, then a hydraulic model with a very modest $10 \mathrm{~cm}$ diameter would need to be on the order of $50 \mathrm{~m}$ long in order to reproduce the process. The experimental study performed by Liu et al. (2020) only had an experimental setup $\sim 10 \mathrm{~m}$ long and this probably partially explains why the laboratory results are somewhat inconsistent with the associated video. Although it is customary to consider limitations due to fluid viscosity in small scale models, the essential problem in a physical model is to properly recreate the behavior of what in some videos appears to be a fine mist where the effect of surface tension would play an important role in determining the size of water droplets. In much the same fashion as viscous effects are overemphasized in a smaller scale model using Froude similarity criteria, so are the effects of surface tension (Olson and Wright 1990, p. 290). These inherent limitations imply that laboratory experiments may continue to be a search for elusive understanding; representative experiments will remain to be conducted and it will be imperative to construct reasonable hypotheses in order that experiments will inform understanding but verification will only indirectly be possible.

It appears that similar limitations are imposed on the use of computational fluid dynamics models to inform the debate. Even if it is accepted that such models can inform problems such as air entraining vortices at pump intakes as an example, at least that problem involves a fairly continuous liquid phase with local penetration by gas while the sewer geyser seems to consist of a very small liquid phase concentration that is dominated by the gas dynamics, a condition that cannot be properly represented in currently available CFD modeling tools. Just like the experimental approach, it will probably be necessary to view CFD modeling as informing understanding and falling short in terms of verification.

What are the messages from the review of available videos with incomplete knowledge of system configuration and flow conditions when combined with the few nuggets of more detailed information that are available? One clear impression is that it is likely that there are myriad specific circumstances that result in geyser formation and it is unlikely that a single explanation will suffice to cover all circumstances. However, it seems that there are some shared characteristics:

- It should be clear that a system must be surcharged with a confined or trapped pocket of air being released through a vertical shaft. Previous experiments indicate that the strength of that rise is greater when the riser diameter is smaller than the nearly horizontal conduit from which the air is released and in the absence of contrary evidence, this observation is likely to still be valid.

- Mechanisms by which the trapped air pockets are formed are probably variable. The geyser is primarily the manifestation of the release of the entire volume of trapped air in a flowing system as opposed to liquid being pushed ahead of a rising air intrusion and this is the main departure from previous interpretations.

- There appears to be a requirement for the liquid phase to exist in a state where it can be readily entrained into the rising air and this has not been adequately recognized in past studies.

One can consider a variety of possible mechanisms for satisfying this last criterion. For example, two phase flows in pipes can be classified according to their general nature (e.g. Mandhane et al. 1974) with flow classes such as slug flow or churn flow involving nonhomogeneous distributions of gas and liquid phases in the direction of flow. If a horizontal pipe is flowing in a nearly full free surface state, waves generated along the interface due to air velocity, which are often referred to as Kelvin-Helmholtz waves, may trap isolated pockets of air along the pipe crown, generating a slug flow. This phenomenon has been suggested to contribute to geyser formation by Leon et al. (2019). However, this process would result in multiple small air volumes associated with each surge in the riser and is not consistent with the bulk of observations of actual sewer geysers as discussed above. Other mechanisms are potentially more reasonable. It is known that some geysers are observed in systems in which the vertical riser acts as a dropshaft with water falling from a higher elevation. This falling water devolves into an air-water mixture which could then be readily entrained in a rapidly rising volume of air. In fact, this is known to be a problem in the function of simple dropshafts and it has required a number of innovations in dropshaft design to avoid this problem. A second potential mechanism is indicated in the study by Liu et al. (2020), where water falling in a junction chamber under certain conditions can create an air-water froth that could then be also be entrained by air released through vertical shafts attached to the junction chamber. It should be pointed out, however, that the above mentioned study only produced vertical surges with periods in the order of $1 \mathrm{~s}$ that with a scale ratio of 1:20 would correspond to $a<5$ s period in the prototype. A video for the same system shows a geyser that lasted $>1 \mathrm{~min}$ so there is an inconsistency in timing that is probably due to the short length of horizontal conduit created in the physical model.

\subsection{Proposed experiments}

It does not seem that there is a straightforward approach to studying this phenomenon and it is necessary to return to the idea of developing a concept and then devising an experiment that can inform that concept. This is not an ideal approach but considering the limitations of small scale hydraulic models of even locally relevant portions of systems and the apparent inability of CFD models to inform important processes, there seems little choice. The experimental configuration presented in Figure 2 is very simple and presents an opportunity if suitable modifications to the experimental apparatus are devised to address the observations described in the previous section. Figure 3 presents a schematic of the proposed modifications. One requirement 
that seems immediately obvious is to increase the volume of air involved in the experiment. Previous choices were primarily based on the idea that it was only necessary to get air to the riser in sufficient volume to create a rapid rise. The experiments by Wright (2013) indicate that the initial rise is a function of air volume but tended to approach a constant value for $V_{\text {air }} / D^{3}>\sim 10$, where $V_{\text {air }}$ is the volume of the air pocket and $D$ is the horizontal conduit diameter. Therefore, the air volume should be increased substantially above that level, probably to where the air volume is equal to or exceeds the water volume in the experiment. It would easy to construct a system where the above ratio is in the order of $\geq 100$, which should be sufficient to develop what could appear as a maintained geyser. The most critical modification is to create a situation where water droplets for an escaping volume of air to interact with can be made to form. It is not clear how to accomplish this situation nor is it clear that surface tension effects will not impact the results so there may need to be resolution with a trial-and-error approach. One method to attempt this is to install a section of the vertical riser near the top that has a set of small diameter holes drilled through the wall permitting inflow from an external reservoir into the riser. Presumably this will mimic the effect of inflow through a dropshaft and, by separating the inflow into many small streams, will enhance the water breakup sufficiently that the interaction with the rising air stream is extensive. There are many variables that could be controlled in this type of setup including the size and number of individual injection points and the total rate of water injection. There are some obvious starting points, however, such as the consideration that the water inflow rate should be a small percentage of the total expected air efflux rate, a quantity that can be estimated with some certainty from existing theories on air intrusions into horizontal conduits (Benjamin 1968). Another potential problem is that surface tension will force the water droplet size to be larger than would be scaled from a prototype system, and this may be a fundamental limitation to performing a small scale experiment. Since the water droplets will primarily arise from the injected water, a surfactant can be added if necessary to decrease the droplet size somewhat. A remaining variable that can be adjusted to control the experimental response is the surcharge level in the system. Wright (2013) showed that increasing the reservoir head on a static system would increase the height of the initial rise, an expected result since the pressure difference across the riser will determine the air velocity once the rising air essentially evacuates the riser. Inspection of data for the Minnesota geyser confirms this same response. Increasing the initial surcharge level will increase the ability or air to transport water droplets upwards.

Success will be defined if it can be demonstrated that the air-water mixture can be lifted through the riser for the duration of the time it takes for the air to evacuate through the riser. Familiarity with the previous experimental apparatus provides some confidence that this configuration will lead to positive results. It is quite possible that alternate experimental configurations could also be used to demonstrate the same outcome.

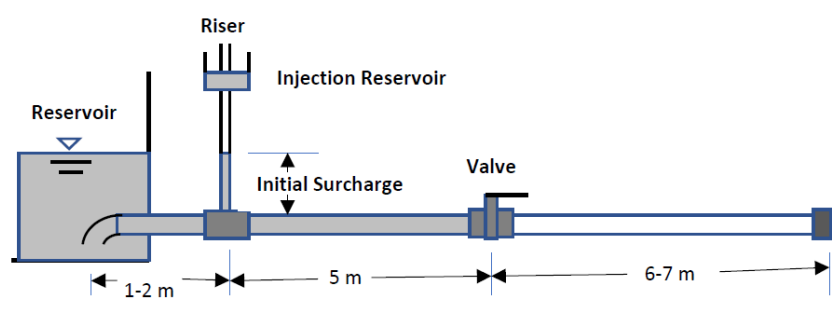

Figure 3 Revised apparatus to show altered configuration.

\section{Conclusions}

Preliminary laboratory experiments to demonstrate sewer geysering were somewhat hampered by a lack of detailed measurements of what occurs in actual systems experiencing the phenomenon and due to logistical constraints in small scale hydraulic modeling of both the geometry and dynamics of a much larger system. In spite of these constraints, some progress has been made in understanding important processes while relevant information has slowly become available. This manuscript summarizes this progress and suggests that the initial view of geyser occurrence was somewhat simplistic and inconsistent with the available evidence. A new experimental study is proposed that should allow the resolution of these inconsistencies. A takeaway lesson is that experiments devised to confirm a concept may do that without actually resolving the original questions if the system to be studied is not adequately understood.

A collection of sewer geyser videos that were mostly obtained by chance and were seldom optimal for the extraction of information were examined. By obtaining many of these, some suggestions as to the nature of the geyser process have been deduced:

- Sewer geysers, as considered in this study, are created by the interaction of water and a relatively large volume of air that has become trapped in a nearly horizontal conduit that is surcharged.

- In order for the mixture to form, the liquid phase needs to be in a form where it can be readily incorporated into the air escaping through a vertical shaft. One mechanism for this appears to be when the shaft acts as a simple dropshaft conveying water from a higher elevation to an underlying conduit.

- The geyser will last for the bulk of the time during which air escapes through the shaft. In many systems evaluated, the duration of the geyser may exceed one minute, a result inconsistent with any laboratory experiment performed to date.

- It is likely that a variety of different sewer configurations could incorporate these features and no one set of circumstances will control the formation of all sewer geysers. A potential configuration that somewhat represents key aspects of some known systems is described. 
With the level of understanding that accompanies the above tentative conclusions, it is possible to return to some of the previous laboratory investigations into sewer geysers and make key alterations in the experimental setup that should, for the first time, demonstrate a sewer geyser created in the laboratory that displays the essential characteristics of those observed in the field.

\section{References}

Benjamin, T.B. 1968. "Gravity Currents and Related Phenomena." Journal of Fluid Mechanics 31 (2): 209-48. https://doi.org/10.1017/S0022112068000133

Burridge, H., and G. Hunt. 2013. "The Rhythm of Fountains: The Length and Time Scales of Rise Height Fluctuations at Low and High Froude Numbers." Journal of Fluid Mechanics 728: 91-119. https://doi.org/10.1017/jfm.2013.263

Cardle J.A., and C.C.S. Song. 1988. "Mathematical Modeling of Unsteady Flow in Storm Sewers." International Journal of Engineering Fluid Mechanics 1 (4): 495-518.

Cataño-Lopera, Y., T.E. Tokyay, J.E. Martin, A.R. Schmidt, R. Lanyon, K. Fitzpatrick, C.F. Scalise, and M.H. Garcia. 2014. “Modeling of a Transient Event in the Tunnel and Reservoir Plan System in Chicago Illinois." Journal of Hydraulic Engineering 140 (9): 05014005. https://doi.org/10.1061/(ASCE)HY.1943-7900.0000888

Chan, S.N., J. Cong, and J.H.W. Lee. 2018. “3D Numerical Modeling of Geyser Formation by Release of Entrapped Air from Horizontal Pipe into Vertical Shaft." Journal of Hydraulic Engineering 144 (3): 04017071. https://doi.org/10.1061/(ASCE)HY.1943-7900.0001416

Davies, R.M., and G.I. Taylor. 1950. “The Mechanics of Large Bubbles Rising Through Extended Liquids and Through Liquids in Vertical Tubes Filled with Water." Proceedings Royal Society of London A 200: 375-90. https://doi.org/10.1098/rspa.1950.0023

Guo, Q. 1989. "Geysering in Urban Storm Drainage Systems." In

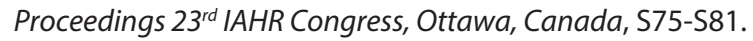

Guo, Q., and C.C.S. Song. 1990. "Surging in Urban Storm Drainage Systems." Journal of Hydraulic Engineering 116 (12): 1523-37. https://doi.org/10.1061/(ASCE)07339429(1990)116:12(1523)

Guo, Q., and C.C.S. Song. 1991. “Dropshaft Hydrodynamics under Transient Conditions." Journal of Hydraulic Engineering 117 (8): 1042-55.

https://doi.org/10.1061/(ASCE)0733-9429(1991)117:8(1042)

Huang, B., S. Wu, and D.Z. Zhu. 2017. "Alleviating Geysers through Standpipes in Sewer Systems." In ASCE World Environmental and Water Resources Congress, 2017, 67-81. https://doi.org.proxy.lib.umich.edu/10.1061/ 9780784480632.005
Huang, B., S. Wu, D.Z. Zhu, and H.E. Schulz. 2017. “Experimental Study of Geysers Through a Vent Pipe Connected to Flowing Sewers" Water Science and Technology 1: 66-76. https://doi.org/10.2166/wst.2018.085

Leon, A.S., S.E. Ibrahem, and Y. Tang. 2019. “An Experimental Study on Violent Geysers in Vertical Pipes." Journal of Hydraulic Research 57 (3): 283-94. https://doi.org/10.1080/00221686.2018.1494052

Leon, A.S., N. Oberg, A.R. Schmidt, and M.H. Garcia. 2011. "Illinois Transient Model: Simulating the Flow Dynamics in Combined Storm Sewer Systems." Journal of Water Management Modeling 19: R241-02. https://doi.org/10.14796/JWMM.R241-02

Lewis, J.W., and S.J. Wright. 2012. "Air-Water Interactions that Generate Large Water Lifts through Vertical Shafts in Storm-water Conduits." Journal of Water Management Modeling 20: R245-02. https://doi.org/10.14796/JWMM.R245-02

Liu, L., W. Shao, and D.Z. Zhu. 2020. "Experimental Study on Stormwater Geyser in Vertical Shaft above Junction Chamber." Journal of Hydraulic Engineering 146 (2): 04019055. https://doi.org/10.1061/(ASCE)HY.1943-7900.0001660

Mandhane, J.M., G.A. Gregory, and K. Aziz. 1974. "A Flow Pattern Map for Gas-Liquid Flow in Horizontal Pipes." International Journal of Multiphase Flow 1 (4): 57-553. https://doi-org.proxy.lib.umich.edu/10.1016/03019322(74)90006-8

Muller, K.Z., J. Wang, and J.G. Vasconcelos. 2017. “Water Displacement in Shafts and Geysering by Uncontrolled Air Pocket Releases." Journal of Hydraulic Engineering 143 (10): 04017043. https://doi.org/10.1061/(ASCE)HY.1943-7900.0001362

Olson, R.M., and S.J. Wright. 1990. Essentials of Engineering Fluid Mechanics. New York: Harper and Row.

Qian, Y., D.Z. Zhu, L. Liu, W. Shao, S. Edwini-Bonsu, and F. Zhou. 2020. "Numerical and Experimental Study on Mitigation of Stormwater Geyser in Edmonton Alberta." Journal of Hydraulic Engineering 146 (3): 04019069. https://doi.org/10.1061/(ASCE)HY.1943-7900.0001684

Shao, Z.S., and S.A. Yost. 2018. "Numerical Investigation of Driving Forces in a Geyser Event Using a Dynamic Multi-phase Navier Stokes Model." Engineering Applications of Computational Fluid Mechanics 12 (1): 493-505. https://doi.org/10.1080/19942060.2018.1459322

Vasconcelos, J.G., and S.J. Wright. 2011. “Geysering Generated by Large Air Pockets Released through Water-filled Ventilation Shafts." Journal of Hydraulic Engineering 137 (5): 543-55. https://doi.org/10.1061/(ASCE)HY.1943-7900.0000332

Wang, X., S. Qian, and H. Chen. 2019. “Experimental Study on Geysers Induced by the Release of Trapped Air in Storage Tunnel Systems." Applied Sciences 9 (24): 5326. https://doi.org/10.3390/app9245326 
Wright, S.J. 2013. “Influence of Air Pocket Volume on Manhole Surge." Journal of Water Management Modeling 21: R246-09. https://doi.org/10.14796/JWMM.R246-09

Wright, S.J., J.W. Lewis, and J.G. Vasconcelos. 2007. “Mechanisms for Stormwater Surges in Vertical Shafts." Journal of Water Management Modeling 15: R227-05. https://doi.org/10.14796/JWMM.R227-05

Wright, S.J., J.W. Lewis, and J.G. Vasconcelos. 2011a. “Geysering in Rapidly Filling Stormwater Tunnels." Journal of Hydraulic Engineering 137 (1): 112-5. https://doi.org/10.1061/(ASCE)HY.1943-7900.0000245
Wright, S.J., J.W. Lewis, and J.G. Vasconcelos. 2011b. "Physical Processes Resulting in Geysering in Rapidly Filling Storm-Water Tunnels." Journal of Irrigation and Drainage Engineering 137 (3): 199-202. https://doi.org/10.1061/(ASCE)IR.1943-4774.0000176

Wright, S.J., J.G. Vasconcelos, and J.W. Lewis. 2017. "Air-Water Interactions in Urban Drainage Systems." Engineering and Computational Mechanics 170 (3): 91-106.

https://doi.org/10.1680/jencm.16.00024 